\title{
Comparing water and paraffin PCM as storage mediums for thermal energy storage applications
}

\author{
Christos Pagkalos ${ }^{1}$, Michalis Gr. Vrachopoulos ${ }^{1, *}$, John Konstantaras $^{1}$, \\ and Kostas Lymperis ${ }^{1}$ \\ ${ }^{1}$ National and Kapodistrian University of Athens, General Department, Energy \\ and Environmental Research Laboratory, 34400, Psachna,Campus, Evia, Greece
}

\begin{abstract}
A CFD analysis is performed in two different heat storage mediums, water and paraffin phase change material (PCM), in order to evaluate and compare the two mediums for use in heating thermal energy storage (HTES) applications. The two mediums use different heat storing mechanisms, namely water uses Sensible Heat Storage, and the PCM Latent heat storage. The applied computational domain represents a single tube of a heat exchanger (HE), and so it comprises of a copper tube with aluminium fins. The geometric characteristics of the domain are taken in accordance with commercially used HE's for HTES applications [1]. The characteristics studied are the stored energy of the system, the temperature of the heat transfer fluid (HTF) in the outlet and the temperature of the storage medium. The results of the simulations showed that for the same mass of storage mediums, the PCM can store more energy than water, for the same temperature of the HTF, as expected. Also, the temperature of the medium for the sensible heat storage rises linearly with the energy stored inside it, while in the latent heat storage mechanism, the temperature of the medium rises linearly till the melting (or solidification) of it, then stays almost steady until the melting of the whole volume and then rises again until it reaches the temperature of the HTF.
\end{abstract}

\section{Introduction}

The environmental sustainability challenge is one of the main concerns for the modern world. Fossil fuel depletion and the environmental pollution are the driving forces for the technologies of the future. One of the ways to achieve environmental sustainability is the use and optimization of renewable energy. In the field of energy saving, energy storage, and in particular heat storage, plays a very important role, and on the other hand, in the field of power supply, energy demand and energy availability often do not coincide in time; therefore, the use of thermal storage system is crucial. There are three main ways for storing thermal energy, sensible, latent and thermo-chemical energy storage [2-4]. In sensible heat storage

\footnotetext{
* Corresponding author: mgrvrachop@uoa.gr
} 
(SHS), thermal energy is stored by raising the temperature of a material, typically solid or liquid. The latent heat storage (LHS), can be achieved using PCMs, i.e. materials characterized by high latent heat of fusion, which through melting or solidification are able to store or provide heat respectively. Finally, thermochemical storage (TCS) is succeeded via a reversible chemical reaction. TCS has the advantage of nearly no losses during heat exchange and a high energy density [2].

In this work, the performance of two different heat storage mediums is evaluated, through CFD analysis. The computational domain considered, is a finned tube, immersed in the storage medium (water or PCM) through which hot (or cold) water flows, in order to charge (or discharge) the medium. The outer walls of the cylinder are considered adiabatic. The fins are used to make the area of the heat transfer larger while the HE is designed taking into account commercially available heat exchanger patterns. The fins are considered to be from aluminum and the tube from copper. The volume of the storage medium depends on the length and the radius of the cylinder as well as the number of fins. The domain represents a single tube inside a heat exchanger and the dimensions respond to the Luvata pattern 1022 [13] one of the commercially used heat exchangers for heating /cooling or Domestic hot water applications.

\section{Sensible and latent heat storage mechanisms}

\subsection{Sensible heat storage}

In Sensible Heat Storage (SHS) systems, the amount of heat stored depends on the specific heat capacity of the medium, the temperature difference from start till the end of the process in the storage medium, and the mass of the medium. Heat from an HTF is transferred through the tube and fins to the storage medium increasing its temperature. The amount of heat stored in or released form a material can be described as:

$$
Q=\int_{T_{i}}^{T_{f}} m \cdot C_{p} \cdot d T
$$

or

$$
Q=m \cdot C_{p} \cdot\left(T_{f}-T_{i}\right)
$$

where $Q[\mathrm{~J}]$ is the amount of thermal energy stored (or released), $T_{i}[\mathrm{~K}]$ is the initial temperature of medium, $T_{f}[\mathrm{~K}]$ is the final temperature of medium, $m[\mathrm{~kg}]$, is the mass of material used to store thermal energy, and $C_{p}[\mathrm{~J} / \mathrm{kgK}]$ is the specific heat capacity of material used to store thermal energy [1] SHS often use solids like stone or brick, or liquids like water, as storage material.

\subsection{Latent heat storage}

Phase change of a certain material can be in the following form: solid-solid, solid-liquid, solid-gas, liquid-gas and vice versa.

In solid-solid transitions, heat is stored as the material is transformed from one crystalline to another. These transitions generally have smaller latent heat and smaller volume changes than solid-liquid transitions. Solid-solid PCMs offer the advantages of less stringent container requirements and greater design flexibility [2] Solid-gas and liquid-gas transition have higher latent heat, but their large volume changes on phase change at (nearly) constant 
pressure, are associated with the containment problems and rule out their potential utility in thermal-storage systems. Large changes in volume make the system complex and impractical [3].

Solid-liquid transformations have comparatively smaller latent heat than liquid-gas. However, these transformations involve only a small change in volume (about $10 \%$ or less). Therefore, solid-liquid transitions have proved to be economically attractive for use in thermal energy storage systems.

Latent heat storage (LHS) is based on the heat absorption or release when a storage material undergoes a phase change from solid to liquid or liquid to gas or vice versa. The storage capacity of the LHS system with a PCM medium is:

$$
Q=\int_{T_{i}}^{T_{m}} m \cdot c_{p, s} \cdot d T+m \cdot \beta \cdot \Delta h_{m}+\int_{T_{m}}^{T_{f}} m \cdot c_{p, l} \cdot d T
$$

or

$$
Q=m \cdot c_{p, s} \cdot\left(T_{m}-T_{i}\right)+\mathrm{m} \cdot \beta \cdot \Delta h_{m}+m \cdot c_{p, l} \cdot\left(T_{f}-T_{i}\right)
$$

where $T_{m}$ is the melting temperature, $c_{p, s}[\mathrm{~J} / \mathrm{kg} \mathrm{K}]$ is the specific heat capacity of solid PCM, $c_{p, l}[\mathrm{~J} / \mathrm{kg} \mathrm{K}]$ is the specific heat capacity of liquid PCM, $\beta$ is the fraction of PCM melted [-], and $\Delta h_{m}[\mathrm{~J} / \mathrm{kg}]$ is the heat of fusion per mass unit [4].

The first term in Eq. (2.4) represents the heat transfer between the storage material and the HTF before the PCM melts. The second term represents the energy produced during phase change and the last term represents the sensible energy transferred between the PCM and the HTF after the PCM melts, until it reaches the inlet water temperature. When the PCM changes phase, a large amount of energy, the latent heat, can be stored or released at an almost constant temperature. Thus, a small difference in temperature can be used for storing and releasing the stored energy. The system with PCM depends on the phase change of the material for capturing and releasing the energy.

PCMs readily and predictably change their phase with a certain input of energy and release this energy at a later time. Compared to the storage of sensible heat, there is almost no temperature variation during the most productive part of the process in the storage [1]. There are a vast number of published materials available for latent heat storage. An overview of the materials and their properties is done, among others, by M. Fatih Demirbas [5].

\section{Problem definition and mathematical modelling}

\subsection{Governing Equations}

The problem of phase change of the paraffin conjugated to conduction in the tube wall and to the forced convection of the HTF is unsteady. The simplifying assumptions, which the mathematical model relies on, for the description of the physics of the problem, are: the PCM is homogeneous and isotropic; natural convection of the PCM is neglected; azimuthal temperature is everywhere negligible (2D solution).

For the simulations, the commercial CFD code, ANSYS FLUENT [12], was used. The model behind the Solidification/Melting Model used by FLUENT is analyzed subsequently. The enthalpy-porosity technique [6-8] is used, for modeling the solidification/melting process. In this technique, the melting interface is not tracked explicitly. Instead, a quantity called the liquid fraction [12], which indicates the fraction of the cell volume that is in liquid form, is associated with each cell in the domain. The liquid fraction is computed at each iteration, based on an enthalpy balance. The mushy zone is a region in which the liquid 
fraction lies between 0 and 1 . The mushy zone is modeled as a "pseudo" porous medium in which the porosity decreases from 1 to 0 as the material solidifies. When the material has fully solidified in a cell, the porosity becomes zero and hence the velocities also drop to zero. More details on the enthalpy-porosity method can be found on the work of Voller and Prakash [8].

For the energy equation, the enthalpy of the material is computed as the sum of the sensible enthalpy, $\mathrm{h}$, and the latent heat, $\Delta \mathrm{H}[12]$ :

$$
\begin{gathered}
H=h+\Delta h \\
\text { where } h=h_{\text {ref }}+\int_{\text {Tref }}^{T} c_{p} d T
\end{gathered}
$$

and $\quad h_{r e f}=$ reference enthalpy $[\mathrm{J} / \mathrm{kg}]$

$$
\begin{aligned}
& T_{\text {ref }}=\text { reference temperature }[\mathrm{K}] \\
& c_{p}=\text { specific heat at constant pressure }[\mathrm{J} / \mathrm{kgK}]
\end{aligned}
$$

The liquid fraction, $\beta$, can be defined as [12]:

$$
\begin{gathered}
\beta=0 \text { if } T<T_{\text {solidus }} \\
\beta=1 \text { if } T>T_{\text {solidus }} \\
\beta=\frac{T-T_{\text {solidus }}}{T_{\text {liquidus }} T_{\text {solidus }}} \text { if } T_{\text {solidus }}<T<T_{\text {liquidus }}
\end{gathered}
$$

Equation 3.3 is referred to as the lever rule.

The latent heat content can now be written in terms of the latent heat of the material, $L$ :

$$
\Delta H=\beta L
$$

For solidification/melting problems, the energy equation is written as:

$$
\frac{\partial}{\partial t}(\rho H)+\nabla \cdot(\rho \vec{v} H)=\nabla \cdot(\kappa \nabla T)+S
$$

where $H=$ enthalpy

$$
\begin{aligned}
& \rho=\text { density } \\
& \vec{v}=\text { fluid velocity } \\
& S=\text { source term }
\end{aligned}
$$

The solution for temperature is essentially an iteration between the energy equation (3.5) and the liquid fraction equation (3.3). Directly use of equation 3.3 [9] to update the liquid fraction usually results in poor convergence of the energy equation. In FLUENT, the method suggested by Voller and Swaminathan [9] is used to update the liquid fraction. For pure metals, where $T_{\text {solidus }} T_{\text {liquidus }}$ are equal, a method based on specific heat, given by Voller and Prakash [8], is used instead.

For the momentum equation, the enthalpy-porosity technique treats the mushy region (partially solidified region) as a porous medium. The porosity in each cell is set equal to the liquid fraction in that cell. In fully solidified regions, the porosity is equal to zero, which 
extinguishes the velocities in these regions. The momentum sink due to the reduced porosity in the mushy zone takes the following form [12]:

$$
S=\frac{(1-\beta)^{2}}{\left(\beta^{3}+\varepsilon\right)} A_{m u s h}\left(\vec{v}-\overrightarrow{v_{p}}\right)
$$

where $\beta$ is the liquid volume fraction, $\varepsilon$ is a small number $(0.001)$ to prevent division by zero, $A_{m u s h}$ is the mushy zone constant, and $\overrightarrow{v_{p}}$ is the solid velocity due to the pulling of solidified material out of the domain (also referred to as the pull velocity).

The mushy zone constant measures the amplitude of the damping; the higher this value, the steeper the transition of the velocity of the material to zero as it solidifies. Very large values may cause the solution to oscillate. The pull velocity is included to account for the movement of the solidified material as it is continuously withdrawn from the domain in continuous casting processes. The presence of this term in Equation 3.6 allows newly solidified material to move at the pull velocity. If solidified material is not being pulled from the domain, $\overrightarrow{v_{p}}=0$.

For some HTF velocities the flow regime becomes turbulent. To overcome this obstacle, sinks are added to all of the turbulence equations in the mushy and solidified zones to account for the presence of solid matter. The sink term is very similar to the momentum sink term (Equation 3.6):

$$
S=\frac{(1-\beta)^{2}}{\left(\beta^{3}+\varepsilon\right)} A_{m u s h} \varphi
$$

where $\varphi$ represents the turbulence quantity being solved $(\kappa, \varepsilon, \omega$, etc.), and the mushy zone constant, $A_{m u s h}$, is the same as the one used in Equation (3.6).

\subsection{Model setup}

Ansys Fluent is the program selected and the main variables investigated are: the energy stored inside the mediums, the outlet HTF temperature and the temperature of the medium. PCMs themselves cannot be used as heat transfer medium. A separate heat transfer medium must be employed with heat exchanger in between to transfer energy from the source to the PCM and from the PCM to the load. The most widely used heat transfer fluid is pure water. Many different fluids have been tried over the years. Propylene glycol-water mix, ethylene glycol water mix, synthetic oil and silicone oil are some examples. [10]. Different flow rates were tested for both laminar and turbulent flow regimes, and indicative results are shown.

The geometry studied, was selected in order to investigate the properties of the storage mediums in use with commercial heat exchangers and the boundary conditions define the process simulated. A $2 \mathrm{~d}$ axisymmetric computational domain was created with the following characteristics shown in table 1.

Table 1. Properties of the Axisymmetric computational domain.

\begin{tabular}{|c|c|}
\hline Circuits configuration & Single tube of $6.6 \mathrm{~m} / 1 \mathrm{~m}$ \\
\hline Inner tube diameter $(\mathrm{mm})$ & 8.6 \\
\hline Outer tube diameter $(\mathrm{mm})$ & 10 \\
\hline Tube material & Copper \\
\hline Fins material & Aluminum \\
\hline Radius of the fins $(\mathrm{mm})$ & 13.8 \\
\hline Fins Pitch $(\mathrm{mm})$ & 5.0 \\
\hline Fins thickness $(\mathrm{mm})$ & 0.3 \\
\hline
\end{tabular}


The mesh was created using Ansys software and the details of it are shown in Figure 1. A mesh independence study was conducted and the mesh selected has 81200 elements for the $1 \mathrm{~m}$ tube and 487200 elements for the $6.6 \mathrm{~m}$ tube.

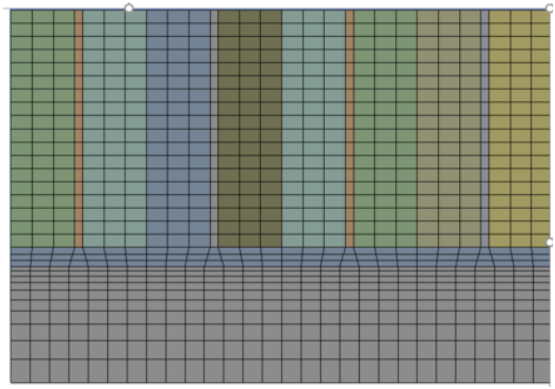

Fig. 1. Computational grid used in the simulations.

For the HTF inlet, a velocity inlet is selected, and for the HTF outlet a pressure outlet. The outer walls are considered adiabatic. The solidification /melting model is enabled and for the mushy zone parameter the default value is selected. The energy model is enabled by default when the solidification/melting model is selected. Finally, both laminar and turbulent models are used for different HTF velocities. The material properties used for the simulations are shown in the table below.

Table 2. Material properties used in the simulations.

\begin{tabular}{|c|c|c|c|c|}
\hline Property & Water & PCM A44 [14] & Copper & Aluminium \\
\hline Thermal conductivity $\boldsymbol{k}[\mathbf{W} / \mathbf{m K}]$ & 0.6 & 0.24 & 387.6 & 218 \\
\hline Density $\boldsymbol{\rho}\left[\mathbf{k g} / \mathbf{m}^{\mathbf{3}}\right]$ & 998.2 & $912(\mathrm{~s}) / 775(1)$ & 8978 & 2719 \\
\hline Specific Heat $\boldsymbol{C}_{\boldsymbol{p}}[\mathbf{J} / \mathbf{k g K}]$ & 4182 & $2400(\mathrm{~s}) / 1800(1)$ & 381 & 871 \\
\hline Viscosity $\boldsymbol{\mu}[\mathrm{kg} / \mathbf{m s}]$ & 0.001003 & $0.007(1)$ & - & - \\
\hline melting Heat of Fusion $[\mathbf{k J} / \mathbf{k g}]$ & - & 250 & - & - \\
\hline solidus temperature $\left[{ }^{\circ} \mathbf{C}\right]$ & - & 42.85 & - & - \\
\hline liquidus temperature $\left[{ }^{\circ} \mathbf{C}\right]$ & - & 45.85 & - & - \\
\hline
\end{tabular}

S-solid; I - liquid

The volume of the storage medium, either water or PCM, is shown in the table below:

Table 3. Heat storage medium volumes.

\begin{tabular}{|c|c|}
\hline Length & Heat Storage Medium volume, $\mathbf{~ m}^{\mathbf{3}}$ \\
\hline $6.6 \mathrm{~m}$ & 0.0058107 \\
\hline $1 \mathrm{~m}$ & 0.0008804 \\
\hline
\end{tabular}

\section{Results and discussion}

Indicative results of the simulations are shown in the following figures. The selected results show a comparison between a HTES using water as storage medium and another using PCM (A44) for two different tube lengths 1 and 6.6 meters and for 30 l/h HTF flow rate. 


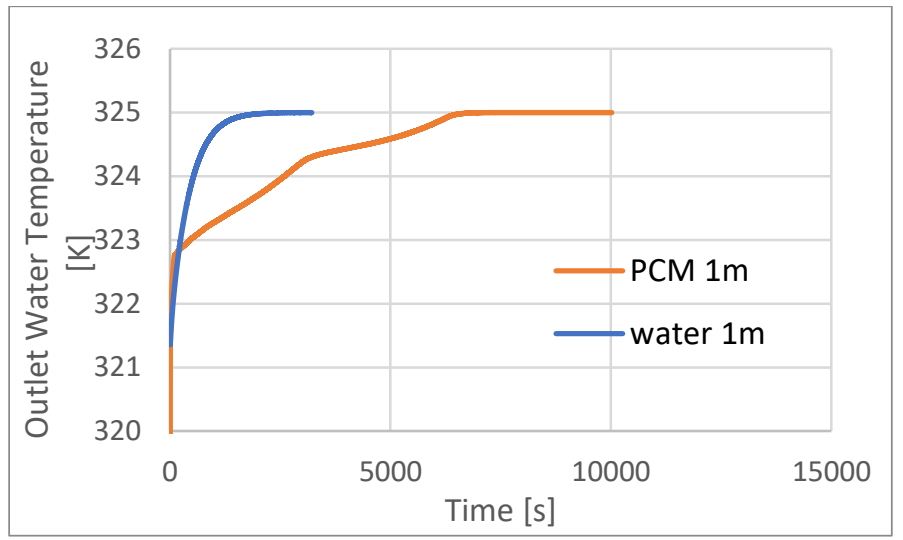

Fig. 2. Outlet water temperature $[\mathrm{K}]$ vs. Time [s] for tube length $1 \mathrm{~m}$.

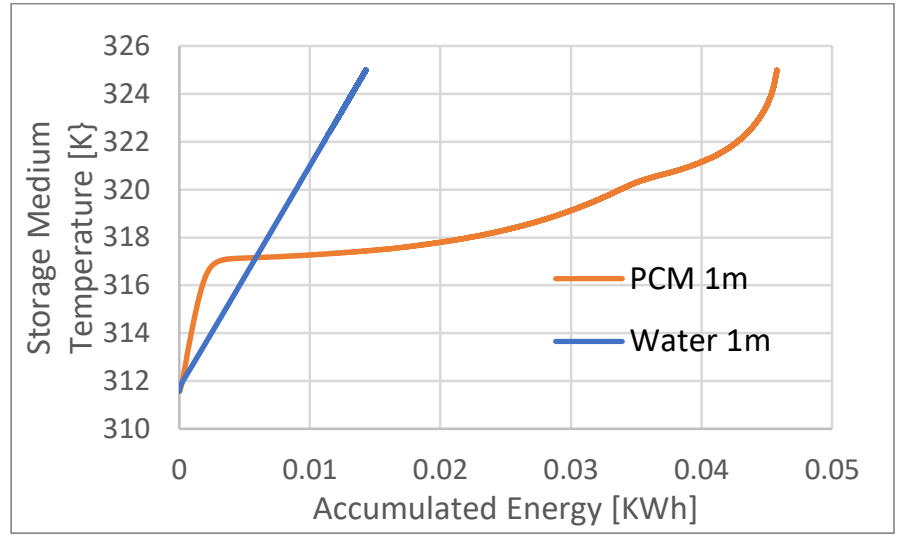

Fig. 3. Storage medium Temperature $[\mathrm{K}]$ vs. Accumulated Energy $[\mathrm{KWh}]$ for tube length $1 \mathrm{~m}$.

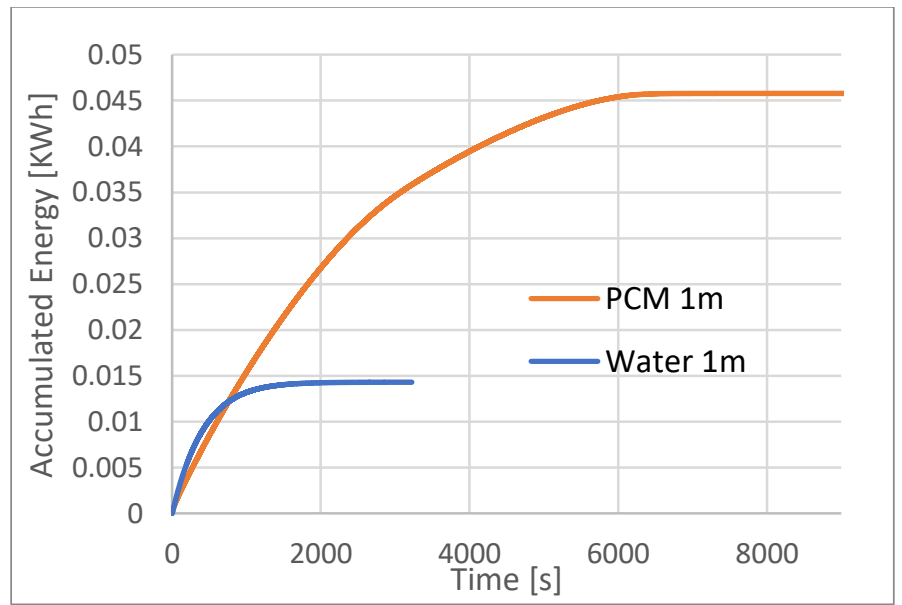

Fig. 4. Accumulated Energy VS Time for tube length $1 \mathrm{~m}$. 


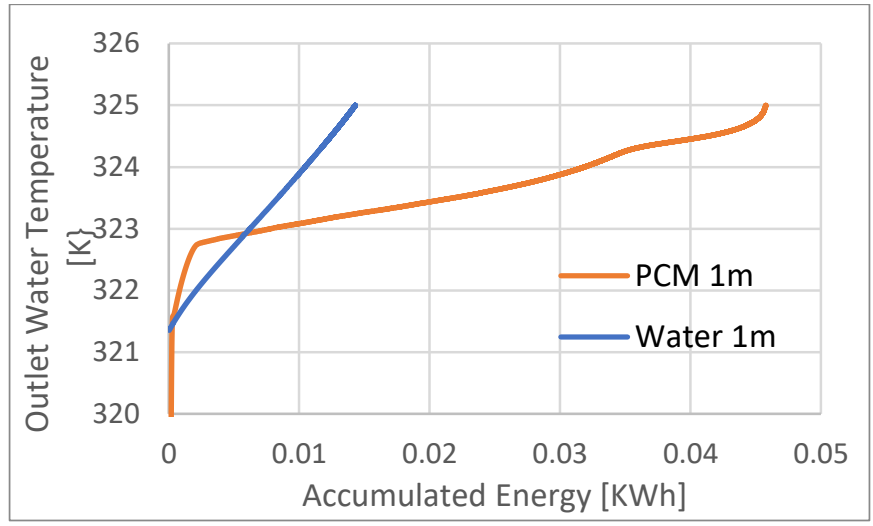

Fig. 5. Outlet Water Temperature [K] VS Accumulated Energy [KWh] for tube length $1 \mathrm{~m}$.

For the 1-meter long cases the storage medium, either water or PCM, occupies $0.0008804 \mathrm{~m}^{3}$, that corresponds to $0.68 \mathrm{~kg}$ in liquid phase, for the PCM and $0.89 \mathrm{~kg}$ for water. The HTF temperature is $325[\mathrm{~K}]\left(51.87^{\circ} \mathrm{C}\right)$ and is kept constant throughout the charging period. The temperature of the fins, tube, and storage medium is considered $312[\mathrm{~K}]\left(39^{\circ} \mathrm{C}\right)$, in order to capture the phenomenon of heat transfer both in the latent and sensible area considering the PCM. For the case of water, only sensible heat is available on this temperature range. The outlet HTF temperature for the case using water as storage medium rises more rapidly than when using PCM and reaches the inlet temperature in faster period of time. The storage medium temperature rises, for the case of water, linearly, while in the PCM case it starts rising linearly then the medium enters the melting phase so latent heat is observed and the gradient is smoother, until all PCM is melted. When all PCM is melted the energy rises again with a greater gradient, because we entered the sensible heat region. The total Accumulated energy is much greater in the PCM case, but the time needed for the full charge is smaller for the water case (Table 4).

Table 4. Indicative results for tube length $1 \mathrm{~m}$.

\begin{tabular}{|c|c|c|}
\hline Tube length 1 m & Water & PCM A44 \\
\hline Accumulated Energy [KWh] & 0.0143 & 0.0467 \\
\hline Time till full charge [s] & 3000 & 8000 \\
\hline
\end{tabular}

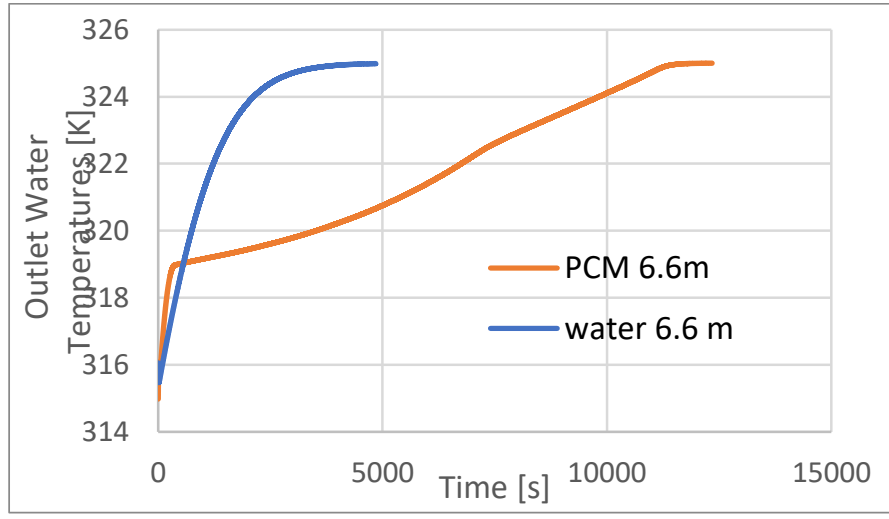

Fig. 6. Outlet water temperature $[\mathrm{K}]$ vs. Time [s] for tube length $6.6 \mathrm{~m}$. 


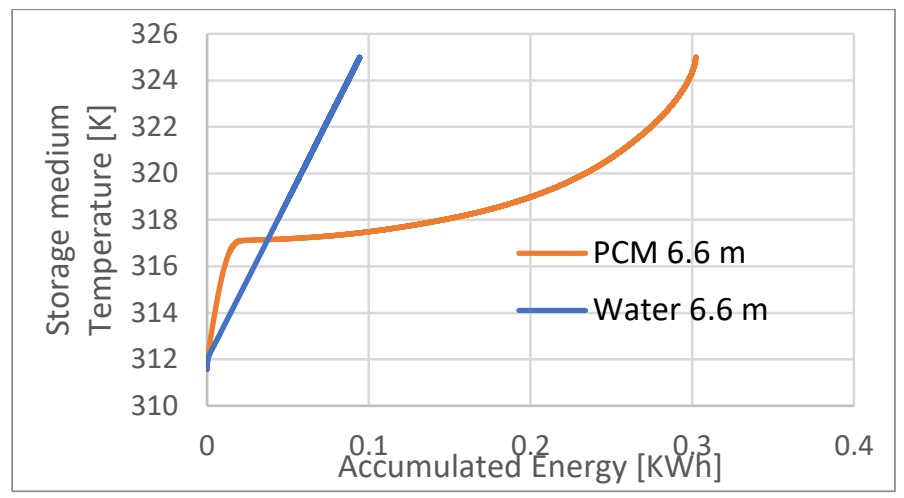

Fig. 7. Storage medium Temperature[K] vs. Accumulated Energy $[\mathrm{KWh}]$ for tube length $6.6 \mathrm{~m}$.

For the 6.6-meter long cases the storage medium, occupies $0.0058107 \mathrm{~m}^{3}$, that corresponds to $4.5 \mathrm{~kg}$ in liquid phase, for the PCM and $5.8 \mathrm{~kg}$ for water. The HTF temperature is $325 \mathrm{~K}\left(51.87^{\circ} \mathrm{C}\right)$ and is kept constant throughout the charging period. The temperature of the fins, tube, and storage medium is considered $312 \mathrm{~K}\left(39^{\circ} \mathrm{C}\right)$, in order to capture the phenomenon of heat transfer both in the latent and sensible areas considering the PCM. For the case of water only sensible heat is available on this temperature range. As in the case of $1 \mathrm{~m}$ tube length, the outlet HTF temperature for the case using water as storage medium, increases rapidly and reaches the inlet HTF temperature in small period of time. For the case of PCM as a storage medium, the time needed for the outlet HTF temperature to reach the inlet HTF temperature is about 3 times slower than the case of water. The total amount of energy stored inside the water is about half of the energy stored inside the PCM. As the storage medium temperature increases the energy stored also increases. For the case of water the temperature increases linearly, from start till end of the process, and for the case of PCM, due to the latent heat present, the temperature increases fairly rapid, but when it reaches $T_{\text {liquidus }}$ the rate of increase, decreases, until all PCM has melted (Table 5).

Table 5. Indicative results for tube length $6.6 \mathrm{~m}$.

\begin{tabular}{|c|c|c|}
\hline Tube length 6.6 m & Water & PCM A44 \\
\hline Accumulated Energy [KWh] & 0.0943 & 0.3022 \\
\hline Time till full charge [s] & 4800 & 12000 \\
\hline
\end{tabular}

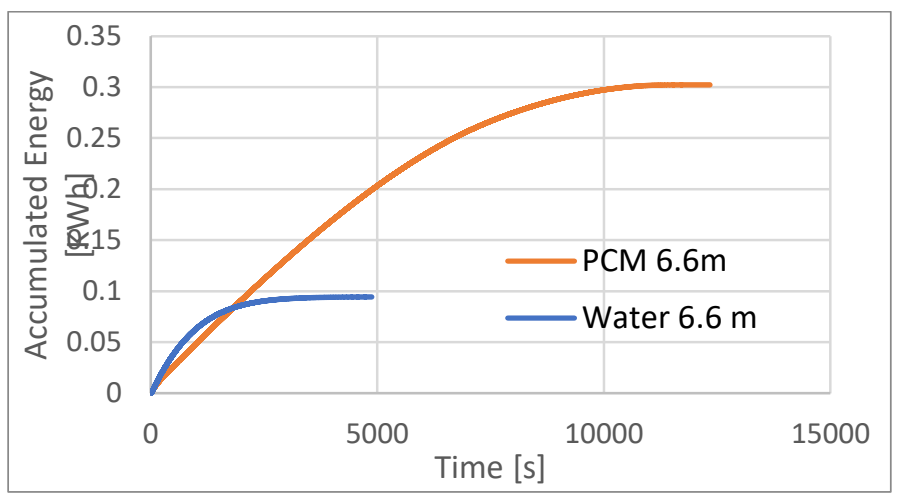

Fig. 8. Accumulated Energy vs. Time for tube length $6.6 \mathrm{~m}$. 


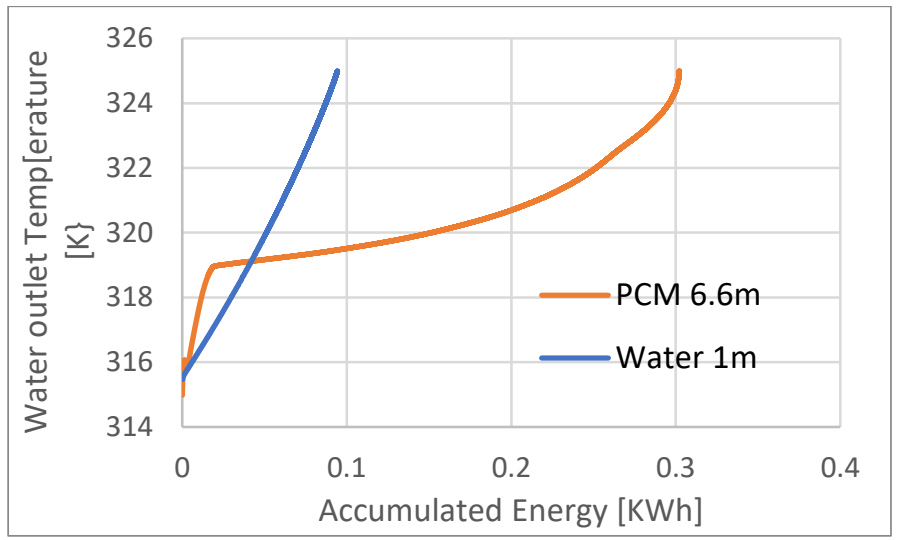

Fig. 9. Outlet Water Temperature $[\mathrm{K}]$ vs. Accumulated Energy $[\mathrm{KWh}]$ for tube length $6.6 \mathrm{~m}$

\section{Conclusions}

In this work a comparison between a sensible heat storage medium (water), and a latent heat storage medium (paraffin PCM A44) is attempted. The study is made on the basis of energy stored in the same material volume under the same charging conditions for comparison purposes. As it was expected using PCM as a storage medium a greater amount of stored energy for the same volume of storage medium, is achieved, but in longer period of time compared to the use of water as storage medium. The PCM system charges about 2 times slower, for both tube lengths, but can store more energy in comparison with the water system.

Sensible heat storage is relatively inexpensive compared to PCM and TCS systems and is applicable to domestic systems, district heating and industrial needs. However, in general, sensible heat storage requires large volumes because of its low energy density (i.e. three to four times lower than that of PCM). Furthermore, sensible heat storage systems require proper design to discharge thermal energy at constant temperatures.

\section{References}

1. M. K. Koukou, M. Gr. Vrachopoulos, N. S. Tachos, G. Dogkas, K. Lymperis, V. Stathopoulos, Thermal Science and Engineering Progress 7, 87 (2018)

2. C. N. Elias, V. N. Stathopoulos, Energy Procedia, in press (2019)

3. A. Abhat, Low temperature latent heat thermal energy storage (In: C. Beghi, Thermal energy storage, Dordrect, Holland, D. Reidel Publication Co., 1981)

4. A. Heinz, H. Schranzhofer, Thermal Energy Storage with Phase Change Materials A Promising Solution? (Institute of Thermal Engineering, Graz University of Technology, Graz)

5. M. F. Demirbas, Energy Sources, Part B: Economics, Planning, and Policy 1, 85 (2006)

6. V. R. Voller, CA, American Metallurgical Society (1987)

7. V. R. Voller, A. D. Brent, K. J. Reid, Technical report. Ranmoor House, Sheffield, England, (1987)

8. V. R. Voller, C. Prakash, Int. J. Heat Mass Transfer 30, 1709 (1987)

9. V. R. Voller, C. R. Swaminathan, Numer. Heat Transfer B 19, 2, 175 (1991) 
10. B. Ramlow, B. Nusz, Solar water heating: a comprehensive guide to solar water and space heating systems (Books for wiser living, Gabriola Island, B.C., New Society Publishers, XV, 2006)

11. IRENA- International Renewable Energy Agency, Thermal Energy Storage Technology Brief, IEA-ETSAP and IRENA(C) Technology Brief E17 (2013)

12. FLUENT 15.0 Theory Guide

13. Luvata coils catgalogue

14. http://www.pcmproducts.net/," PCM Products Ltd., [Online]. Available: http://www.pcmproducts.net/. [Accessed February 2019]. 